
Revue Revue de l'histoire des religions
de I'histoire des religions
$1 \mid 2012$
Judaïsme / christianisme : syncrétismes, antinomies, dissonances

\title{
François TRÉMOLIÈRES, Fénelon et le sublime. \\ Littérature, anthropologie, spiritualité
}

Paris, Honoré Champion («Lumière classique », 86), 2009, 727 p., 24 cm,

$110 €$, ISBN 978-2-7453-2020-9

Sylvio Hermann De Franceschi

\section{OpenEdition}

\section{Journals}

Édition électronique

URL : http://journals.openedition.org/rhr/7878

DOI : $10.4000 /$ rhr.7878

ISSN : 2105-2573

Éditeur

Armand Colin

Édition imprimée

Date de publication : 1 mars 2012

Pagination : 150-153

ISSN : 0035-1423

Référence électronique

Sylvio Hermann De Franceschi, « François trémolı̀eres, Fénelon et le sublime. Littérature, anthropologie, spiritualité ", Revue de l'histoire des religions [En ligne], 1 | 2012, mis en ligne le 04 avril 2012, consulté le 22 septembre 2020. URL : http://journals.openedition.org/rhr/7878; DOI : https://doi.org/10.4000/rhr. 7878

Ce document a été généré automatiquement le 22 septembre 2020.

Tous droits réservés 


\section{François TRÉMOLIÈRES, Fénelon et le sublime. Littérature, anthropologie, spiritualité}

Paris, Honoré Champion («Lumière classique », 86), 2009, 727 p., $24 \mathrm{~cm}$, $110 €$, ISBN 978-2-7453-2020-9

Sylvio Hermann De Franceschi

\section{RÉFÉRENCE}

François TRÉMOLIÈRES, Fénelon et le sublime. Littérature, anthropologie, spiritualité, Paris, Honoré Champion (« Lumière classique », 86), 2009, 727 p., 24 cm, $110 €$, ISBN 978-2-7453-2020-9.

1 Soutenue à l'Université de Paris IV en 2002 sous la direction de Gérard Ferreyrolles, la thèse de doctorat de François Trémolières sur Fénelon et le sublime est sans doute l'une des plus importantes contributions récemment versées au dossier, déjà conséquent, des études féneloniennes. Ouvrage qui se place délibérément au carrefour de la critique littéraire, de l'histoire religieuse, de l'histoire de la philosophie et de la spiritualité, selon une tradition française illustrée par Louis Cognet, Jean Orcibal et surtout Jacques Le Brun, dont les travaux féneloniens ont exercé une manifeste influence - mais il ne pouvait en être autrement - sur la démarche de F. Trémolières.

2 Depuis longtemps, les études françaises d'histoire des idées à l'ưge classique se trouvent face à deux modèles méthodologiques imposants entre lesquels elles sont, pour ainsi dire, sommées de choisir. D'une part, l'approche très historique d'Henri Gouhier, qui, dans ses innombrables publications sur Descartes, Malebranche, Pascal et Fénelon, a toujours cherché à retracer la dynamique de construction et de progressive élaboration des systèmes philosophiques ou théologiques des auteurs auxquels il s'intéressait; d'autre part, la démarche surplombante - presque structuraliste - de Martial Gueroult, qui, dans son Descartes selon l'ordre des raisons (1953) ou dans les trois volumes de son 
Malebranche (1955-1959), a préféré, au contraire, procéder à la restitution rétrospective d'une unité systématique de la pensée des deux philosophes. Entre des voies si différentes, le grand et incontestable mérite du livre de F. T. est d'avoir su proposer une alternative et permettre enfin d'esquisser, à travers une approche par coupe transversale et qui multiplie les dessins de cohérences significatives, un véritable système de Fénelon en partant de la notion cardinale de sublime. L'entreprise était d'autant plus ardue que l'œuvre fénelonienne se caractérise par une extrême variété textuelle: lettres spirituelles, roman, dialogues, correspondances, mandements et instructions pastorales, courts traités philosophiques et théologiques, avis ponctuels. D'extraire une unité d'une telle diversité était un défi : s'imposait alors le recours à un concept essentiel - le sublime - à l'aune duquel essayer de délimiter les grandes dimensions d'un système de pensée qu'il convenait aussi de soigneusement contextualiser.

3 Le choix de la notion de sublime paraissait naturel, tant elle est traditionnellement rapportée à Fénelon; elle avait en outre l'inestimable avantage d'être susceptible d'une application conjointe aux domaines de la rhétorique, de la théologie, de la spiritualité et de la philosophie. Ainsi du problème de l'éloquence sacrée, auquel F. T. consacre des pages très novatrices, relevant que Fénelon modifie la tripartition traditionnelle du docere, mouere, delectare pour substituer peindre à plaire: « La peinture apparaît comme l'instrument d'une nouvelle rhétorique, qui se retrouve dans le sublime longinien en tant que ce dernier exprime à la fois le dépassement des genres, les limites d'une approche technique (catégorielle) de l'éloquence, enfin le souci expressif du naturel » (p.114-115). Dans le genre de l'éloge, Fénelon a obstinément tenu que l'éloquence authentique ne devait pas se réduire au grand style, mais à la simple peinture des faits et à leur restitution variée: "Ce sublime qui n'est employé que quand il faut l'être exprime à la fois la critique morale de la fausse grandeur (comme de la rhétorique qui l'accompagne des phrases magnifiques) et l'idéal poétique de naturel, de simplicité, partant de variété, accordé à la transformation du plaisir en peinture qui le rend acceptable, transparent» (p.129). Au sublime augustinien, Fénelon préférait en définitive le sublime de l'Écriture, et en particulier le sublime johannique, auquel il mesurait sans cesse la valeur de la véritable éloquence du prédicateur chrétien opposé au prédicateur mondain. Pour l'archevêque de Cambrai, la persuasion s'obtient bien plus en s'attachant l'auditoire par un sublime de suavité qu'en l'impressionnant par le sublime fracassant d'une éloquence tonnante : le parallèle fait par F. T. entre Fénelon et Bossuet est ici très éclairant.

4 Passant de l'éloquence et de la prédication à la question, fondamentale, de l'apologétique fénelonienne, F.T. y voit l'expression d'un «sublime de l'incommensurable » et relève que Fénelon s'inscrit à plusieurs égards dans le cadre d'une mentalité typique du Grand Siècle pour qui «il n'est pas d'acte de foi - pas de croyance - sans une part d'irrationnel, c'est-à-dire d'obscurité pour la raison » (p. 198). À travers une minutieuse analyse des textes théologiques de Fénelon relatifs à la querelle janséniste, et notamment la fameuse Instruction pastorale en forme de dialogues du $1^{\mathrm{er}}$ janvier 1714, F. T. montre à l'œuvre chez Fénelon la conscience d'un « sublime métaphysique " par rapport à quoi se fait le départ entre ce que la raison peut appréhender et ce devant quoi elle ne peut faire qu'acte de soumission. L'étude des ouvrages de Fénelon relatifs au jansénisme est également l'occasion de suivre, grû́ce aux abondantes considérations consacrées à la notion janséniste de délectation victorieuse, l'élaboration de la conception fénelonienne du delectare, l'un des trois piliers 
de la rhétorique classique. À partir de quoi F. T. peut aborder l'examen des textes de Fénelon qui prennent part à la controverse sur le quiétisme et qui sont le lieu du principal ensemble d'occurrences du "sublime » en dehors des Dialogues sur l'éloquence et de la Lettre à l'Académie. On passe alors d'un sublime rhétorique et d'un sublime métaphysique à un sublime "pratique » ou expérimental : "Sublime en vient alors à désigner deux choses très différentes, et qui paraissent parfois presque contradictoires [...] : l'expérience mystique dans sa positivité, décrite traditionnellement comme union à Dieu; et sa purification par les modernes, qui ont pris soin de reconnaître dans l'expression fautive de fond de l'û́me seulement la pure volonté»(p. 427). Il y a, chez Fénelon, une très habile utilisation de l'ambivalence même du sublime : le terme, sur un plan exotérique, se rapporte à l'élévation mystique, et sur un plan ésotérique, à l'indicible de l'expérience surnaturelle. Double sublime qui est mis au service de la souplesse persuasive de Fénelon, pourtant mise en échec lors de ses tractations avec la curie romaine.

5 Au fil de ses analyses, F. T. donne à voir l'extraordinaire complexité d'une notion à partir de quoi peut se ressaisir une compréhension unifiée de la diversité de l'œuvre de Fénelon: "Dans le corpus fénelonien, le sublime appartient à la fois au domaine du profane avec la rhétorique, et [...] au domaine du sacré avec la spiritualité; ce non seulement à leur intersection : l'éloquence de la chaire [...] ; mais aussi et séparément sur leur terrain propre: celui d'une esthétique et d'une poétique [...], celui de la mystique et de la spiritualité » (p. 453). Élargissant son enquête aux textes politiques et moraux de Fénelon, et en particulier au Télémaque, F. T. est ainsi conduit à distinguer trois domaines d'emploi du sublime fénelonien: «L'usage rhétorique, où l'originalité de Fénelon écrivain, son élaboration d'une poétique et d'une stylistique de la simplicité chargée des harmoniques de sa spiritualité [...], rencontrent une attente collective qui lui fait investir le champ de la mondanité ; l'usage que nous qualifierions volontiers de critique, dans le domaine de la spiritualité elle-même, terme convenu de la contemplation la plus sublime, dont la fin du siècle a appris à se méfier, et que l'épreuve [...] purifie; enfin, dans le Télémaque, un goût sublime de la vérité et de la vertu, pour qualifier l'expérience béatifique» (p.637). Insistant, en conclusion, sur le projet résolument monographique qui a commandé l'élaboration de sa recherche, François Trémolières en explicite aussi l'immense vertu, qui consiste à proposer au lecteur la reconstitution, par touches successives et par quête de cohérences apparemment lointaines mais en réalité très proches, d'un véritable système de Fénelon. Sans croire un seul instant avoir rendu compte de l'ensemble des résultats apportés par un livre aussi riche et aussi dense, on espère seulement en avoir pointé le prix singulier et l'apport méthodologique incontestable.

\section{AUTEURS}

\section{SYLVIO HERMANN DE FRANCESCHI}

Université de Limoges. 